\title{
TRANSFER IDEALS AND TORSION IN THE MORAVA E-THEORY OF ABELIAN GROUPS
}

\author{
TOBIAS BARTHEL AND NATHANIEL STAPLETON
}

\begin{abstract}
Let $A$ be a finite abelian $p$ group of rank at least 2 . We show that $E^{0}(B A) / I_{t r}$, the quotient of the Morava $E$-cohomology of $A$ by the ideal generated by the image of the transfers along all proper subgroups, contains $p$-torsion. The proof makes use of transchromatic character theory.
\end{abstract}

\section{INTRODUCTION}

The close relationship between the Morava $E$-cohomology of spaces and algebraic geometry has played an important role in problems related to power operations for Morava $E$-theory, character theory for Morava $E$-theory, and understanding $H_{\infty}$-ring maps between $H_{\infty}$-ring spectra and $E$. Let $\mathbb{G}$ be the universal deformation formal group associated to $E$ and write $I_{t r}$ for the transfer ideal. A fundamental result of Strickland's in [Str98] shows that $E^{0}\left(B \Sigma_{p^{k}}\right) / I_{t r}$ corepresents the formal scheme parametrizing subgroup schemes of order $p^{k}$ in $\mathbb{G}$. For instance, this theorem plays a vital role in Rezk's study [Rez09, Rez12] of the Dyer-Lashof algebra for Morava $E$-theory. One of the key ingredients in Strickland's proof is the fact that $E^{0}\left(B \Sigma_{p^{k}}\right) / I_{t r}$ is a free $E^{0}$-module.

Let Level $\left(A^{*}, \mathbb{G}\right)$ be the formal scheme of $A^{*}$-level structures in $\mathbb{G}$, where $A^{*}$ is the Pontryagin dual of a finite abelian $p$-group $A$. In [Dri74], it is shown that the ring of functions on $\operatorname{Level}\left(A^{*}, \mathbb{G}\right)$ is a finitely generated free $E^{0}$-module. Proposition 7.5 in [AHSO4] implies that there is a close relationship between $E^{0}(B A) / I_{t r}$ and the ring of functions on $\operatorname{Level}\left(A^{*}, \mathbb{G}\right)$. In fact, if we let $\left(E^{0}(B A) / I_{t r}\right)^{\text {free }}$ be the image of $E^{0}(B A) / I_{t r}$ in $\mathbb{Q} \otimes E^{0}(B A) / I_{t r}$, then there is a canonical isomorphism

$$
\operatorname{Spf}\left(\left(E^{0}(B A) / I_{t r}\right)^{\text {free }}\right) \cong \operatorname{Level}\left(A^{*}, \mathbb{G}\right) .
$$

For a transitive abelian subgroup $A \subseteq \Sigma_{p^{k}}$, Ando, Hopkins, and Strickland use this isomorphism to give an algebro-geometric description of the power operation

$$
E^{0} \stackrel{P_{p^{k}} / I_{t r}}{\longrightarrow} E^{0}\left(B \Sigma_{p^{k}}\right) / I_{t r} \rightarrow E^{0}(B A) / I_{t r} \rightarrow\left(E^{0}(B A) / I_{t r}\right)^{\text {free }} .
$$

This motivates the question of whether $E^{0}(B A) / I_{t r}$ contains $p$-torsion, i.e., if

$$
\left(E^{0}(B A) / I_{t r}\right)^{\text {free }} \nsucceq E^{0}(B A) / I_{t r} .
$$

These two rings are isomorphic when $A$ is cyclic. In contrast, the main result of this note implies that they are distinct when the rank of $A$ is greater than 1 .

Theorem (Theorem 3.1). Let $A$ be a finite abelian p-group with $\operatorname{rank}(A) \geq 2$. Let $I_{t r} \subset E^{0}(B A)$ be the ideal generated by the image of transfers along all proper subgroups of $A$. The $E^{0}$-algebra $E^{0}(B A) / I_{\text {tr }}$ contains p-torsion.

Date: March 2, 2020.

2010 Mathematics Subject Classification. 55N22 (primary); 55P42, 55S25 (secondary).

The authors would like to thank the Max Planck Institute for Mathematics for its hospitality and Jeremy Hahn for helpful conversations. The second author was supported by NSF grant DMS-1906236. 
In particular, this answers a question raised in [HS20, Remark 5.3]. Our proof uses the transchromatic character maps of [BS16] to first reduce to a question about $p$-adic $K$-theory. We then use classical character theory to show that $K_{p}^{0}(B A) / I_{t r}$ is non-zero and contains torsion when $\operatorname{rank}(A) \geq 2$.

\section{2. $p$-ADIC $K$-THEORY OF FINITE ABELIAN $p$-GROUPS AND TORSION}

Fix a prime $p$ and let $K_{p}$ denote $p$-adic $K$-theory. Given a finite $p$-group $G$, there is a canonical isomorphism

$$
\mathbb{Z}_{p} \otimes R(G) \stackrel{\cong}{\rightrightarrows} K_{p}^{0}(B G),
$$

where $R(G)$ is the representation ring of $G$. It follows that there is an isomorphism

$$
K_{p}^{0}\left(B \mathbb{Z} / p^{k}\right) \cong \mathbb{Z}_{p}[x] /\left(x^{p^{k}}-1\right) \cong \mathbb{Z}_{p}[x] /\left(\left[p^{k}\right](x)\right),
$$

where $\left[p^{k}\right](x)$ is the $p^{k}$-series for the multiplicative formal group law. Since $R(G \times H) \cong R(G) \otimes$ $R(H)$ for finite groups $G$ and $H$, the isomorphisms above give an explicit description of $K_{p}^{0}(B A)$ for any finite abelian $p$-groups $A$.

Character theory for $p$-adic $K$-theory was developed by Adams in [Ada78, Section 2] and generalized to all Morava $E$-theories by Hopkins, Kuhn, and Ravenel in [HKR00]. Let $D=$ $\operatorname{colim}_{i \geq 0} \mathbb{Z}_{p}\left(\zeta_{p^{i}}\right)$, the ring obtained from $\mathbb{Z}_{p}$ by adjoining all of the $p^{i}$ th roots of unity as $i$ varies. Let $C_{0}=\mathbb{Q} \otimes D$. For a finite group $G$, the character map is a canonical inclusion of $\mathbb{Z}_{p}$-algebras

$$
K_{p}^{0}(B G) \hookrightarrow C l_{p}\left(G, C_{0}\right),
$$

where $C l_{p}\left(G, C_{0}\right)$ is the ring of $C_{0}$-valued functions on the set of conjugacy classes of $p$-power order elements in $G$. By construction, the character map factors through $C l_{p}(G, D)$, the ring of $D$-valued functions on the set of conjugacy classes of $p$-power order elements in $G$, giving

$$
K_{p}^{0}(B G) \hookrightarrow C l_{p}(G, D) \hookrightarrow C l_{p}\left(G, C_{0}\right) .
$$

The ring $D$ is local with maximal ideal $(p) \subset D$.

Given $H \subseteq G$, there is a transfer map $K_{p}^{0}(B H) \rightarrow K_{p}^{0}(B G)$, which is a map of modules for the $K_{p}^{0}(B G)$-module structure coming from restriction. This transfer map is compatible with the transfer map on class functions

$$
\operatorname{Tr}_{H}^{G}: C l_{p}\left(H, C_{0}\right) \rightarrow C l_{p}\left(G, C_{0}\right)
$$

given by

$$
\operatorname{Tr}_{H}^{G}(f)([g])=\frac{1}{|H|} \sum_{k \in G, k g k^{-1} \in H} f\left(k g k^{-1}\right),
$$

see for example [HKR00, Theorem D]. Given a finite abelian $p$-group $A$ and a subgroup $A^{\prime} \subseteq A$, the transfer map $K_{p}^{0}\left(B A^{\prime}\right) \rightarrow K_{p}^{0}(B A)$ is determined by the image of $1 \in K_{p}^{0}\left(B A^{\prime}\right)$ as the restriction $K_{p}^{0}(B A) \rightarrow K_{p}^{0}\left(B A^{\prime}\right)$ is surjective. Applying the formula above, we have

$$
\operatorname{Tr}_{A^{\prime}}^{A}(1)(a)= \begin{cases}\left|A / A^{\prime}\right| & \text { if } a \in A^{\prime} \\ 0 & \text { otherwise. }\end{cases}
$$

We define $I_{t r} \subset K_{p}^{0}(B A)$ to be the ideal generated by the image of the transfer maps along all proper subgroups of $A$. We will abuse notation and denote the ideal generated by transfers along all proper subgroups in $C l_{p}\left(A, C_{0}\right)$ by $I_{t r}$ as well. In fact, since the class functions in (2.2) take values in the integers, we may also consider the ideal $I_{t r}$ in $C l_{p}(A, D)$.

Lemma 2.3. Assume $A$ is a finite abelian p-group, then the commutative ring $K_{p}^{0}(B A) / I_{t r}$ is non-zero. 
Proof. This is clear when $A$ is cyclic. Now we assume that $\operatorname{rank}(A) \geq 2$. Since there is a map of commutative rings

$$
K_{p}^{0}(B A) / I_{t r} \rightarrow C l_{p}(A, D) / I_{t r} \cong\left(\prod_{a \in A} D\right) / I_{t r},
$$

it suffices to prove that the target is non-zero. Consider the factor of $\prod_{a \in A} D$ corresponding to $0 \in A$. By the formula in (2.2), the projection of $I_{t r}$ to that factor is contained in the ideal $(p) \subset D$.

Lemma 2.4. The commutative ring $K_{p}^{0}\left(B(\mathbb{Z} / p)^{\times 2}\right) / I_{\text {tr }}$ is an $\mathbb{F}_{p}$-algebra.

Proof. By Lemma 2.3, it suffices to show that $(p) \subseteq I_{t r}$. Let $V_{1}, \ldots, V_{p+1}$ be the maximal abelian subgroups of $A=(\mathbb{Z} / p)^{\times 2}$, then

$$
p=\left(\sum_{i=1}^{p+1} \operatorname{Tr}_{V_{i}}^{A}(1)\right)-\operatorname{Tr}_{0}^{A}(1) .
$$

This can be checked in the ring of class functions. The value of the class function

$$
\sum_{i=1}^{p+1} \operatorname{Tr}_{V_{i}}^{A}(1)
$$

is $p$ on any non-zero element of $A$ and $p^{2}+p$ on $0 \in A$.

Proposition 2.5. Assume $A$ is a finite abelian p-group with $\operatorname{rank}(A) \geq 2$, then the commutative ring $K_{p}^{0}(B A) / I_{\text {tr }}$ is an $\mathbb{F}_{p}$-algebra.

Proof. Fix a surjection $\rho: A \rightarrow(\mathbb{Z} / p)^{\times 2}$. Since $\rho$ is surjective, given any proper subgroup $H \subset(\mathbb{Z} / p)^{\times 2}, \rho^{-1}(H)$ is a proper subgroup of $A$. Thus the map $\rho$ induces a map of commutative rings

$$
K_{p}^{0}\left(B(\mathbb{Z} / p)^{\times 2}\right) / I_{t r} \rightarrow K_{p}^{0}(B A) / I_{t r} .
$$

It suffices to prove that $K_{p}^{0}\left(B(\mathbb{Z} / p)^{\times 2}\right) / I_{t r}$ is an $\mathbb{F}_{p}$-algebra, which is the content of Lemma 2.4.

\section{Morava $E$-Theory of Finite abelian $p$-Groups AND TORSion}

Let $E$ be a height $n$ Morava $E$-theory at the prime $p$ and let $A$ be a finite abelian $p$-group. Just as before, we define the transfer ideal $I_{t r} \subset E^{0}(B A)$ to be the ideal generated by the image of the transfer maps along proper subgroups of $A$.

Morava $E$-theory admits a variety of versions of character theory (e.g., [HKR00], [GS99], [Sta13], [Sta15], [BS16]). In [BS16, Section 5] the authors constructed a commutative ring $\bar{C}_{1}$, which depends on $n$ and $p$, that is a flat $E^{0}$-algebra and a faithfully flat $\mathbb{Z}_{p}$-algebra with the property that

$$
\bar{C}_{1} \otimes_{E^{0}} E^{0}(B A) \cong \prod_{A \times n-1} \bar{C}_{1} \otimes_{\mathbb{Z}_{p}} K_{p}^{0}(B A) .
$$

In fact, the isomorphism above can be extended to all finite groups but we will not need that generality here. Write $\mathcal{L}$ for the free loop space functor. Since $\mathcal{L}^{n-1} B A \simeq A^{\times n-1} \times B A$, we have an isomorphism

$$
\prod_{A^{\times n-1}} \bar{C}_{1} \otimes_{\mathbb{Z}_{p}} K_{p}^{0}(B A) \cong \bar{C}_{1} \otimes_{\mathbb{Z}_{p}} K_{p}^{0}\left(\mathcal{L}^{n-1} B A\right) .
$$


The isomorphism is compatible with transfers in the sense that there is a commutative diagram of abelian groups

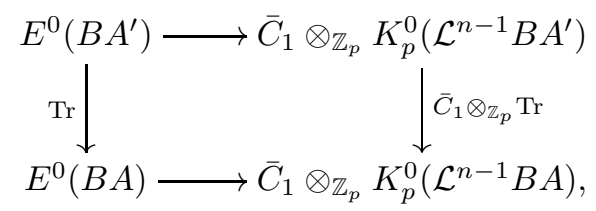

where the transfer on the right is along the finite cover $\mathcal{L}^{n-1} B A^{\prime} \rightarrow \mathcal{L}^{n-1} B A$. The commutativity of this diagram is due to the fact that it is induced by a map of cohomology theories. This is explained in Proposition 4.14 in [BS17].

We say that a commutative ring $R$ contains torsion if the underlying $\mathbb{Z}$-module contains torsion. For a flat map $R \rightarrow S$ of commutative rings, an $R$-module $M$ contains $p$-torsion if $S \otimes_{R} M$ contains $p$-torsion. This follows from the fact that if $p: M \rightarrow M$ is injective then $p: S \otimes_{R} M \rightarrow S \otimes_{R} M$ is injective.

Theorem 3.1. Assume that $A$ is an abelian p-group with $\operatorname{rank}(A) \geq 2$, then the commutative ring $E^{0}(B A) / I_{t r}$ contains p-torsion.

Proof. It follows from Theorem 6.9 in [BS16] that

$$
\bar{C}_{1} \otimes_{E^{0}} E^{0}(B A) / I_{t r} \cong \prod_{\left(a_{i}\right) \in A^{\times n-1}} \bar{C}_{1} \otimes_{\mathbb{Z}_{p}} K_{p}^{0}(B A) / I_{t r}^{\left(a_{i}\right)}
$$

where $I_{t r}^{\left(a_{i}\right)}$ is a certain transfer ideal depending on the tuple $\left(a_{i}\right)$. The transfer ideals in the target were studied in [HS20]. The ideal $I_{t r}^{(0)} \subset K_{p}^{0}(B A)$ is the ideal generated by transfers from all proper subgroups of $A$. Proposition 2.5 implies that $K_{p}^{0}(B A) / I_{t r}^{(0)}$ has torsion. Since $\bar{C}_{1}$ is faithfully flat as a $\mathbb{Z}_{p}$-algebra, the ring

$$
\prod_{\left(a_{i}\right) \in A^{\times n-1}} \bar{C}_{1} \otimes_{\mathbb{Z}_{p}} K_{p}^{0}(B A) / I_{t r}^{\left(a_{i}\right)}
$$

contains torsion, hence so does $\bar{C}_{1} \otimes_{E^{0}} E^{0}(B A) / I_{t r}$. Since $\bar{C}_{1}$ is a flat $E^{0}$-algebra, it follows that $E^{0}(B A) / I_{t r}$ contains torsion as well.

We conclude with a short comparison of Theorem 3.1 with the existing literature. In [Str98], Strickland proves that $E^{0}\left(B \Sigma_{p^{k}}\right) / I_{t r}$ is a free $E^{0}$-module, where $I_{t r}$ is the ideal generated by transfers along $\Sigma_{i} \times \Sigma_{j} \subset \Sigma_{p^{k}}$ with $i+j=p^{k}$ and $i, j>0$. Of course, $\Sigma_{p^{k}}$ is only abelian when $p=2$ and $k=1$, in which case it is cyclic. Further, given a transitive abelian subgroup $A \subseteq \Sigma_{p^{k}}$, there is an induced map of commutative rings

$$
E^{0}\left(B \Sigma_{p^{k}}\right) / I_{t r} \rightarrow E^{0}(B A) / I_{t r} .
$$

However, due to the direction of the map, Theorem 3.1 does not have any consequences on the existence torsion in $E^{0}\left(B \Sigma_{p^{k}}\right) / I_{t r}$.

In [SS15], the main result of [Str98] is generalized to groups of the form $A \nmid \Sigma_{p^{k}}$. The transfer ideal $I_{t r} \subset E^{0}\left(B A \imath \Sigma_{p^{k}}\right)$ considered there is generated by transfers along $A$ 々 $\left(\Sigma_{i} \times \Sigma_{j}\right) \subseteq A \nmid \Sigma_{p^{k}}$ with $i+j=p^{k}$ and $i, j>0$. They show that $E^{0}\left(B A \imath \Sigma_{p^{k}}\right) / I_{t r}$ is a free $E^{0}$-module. In [Nel18], Nelson proves that $E^{0}\left(B \Sigma_{p^{k}} \zeta \Sigma_{p^{l}}\right) / I_{t r}$ is free, where $I_{t r}$ is generated by transfers along the subgroups $\left(\Sigma_{i} \times \Sigma_{j}\right) \imath \Sigma_{p^{l}} \subset \Sigma_{p^{k}}\left\{\Sigma_{p^{l}}\right.$, where $i+j=p^{k}$ and $i, j>0$ and along the subgroups $\Sigma_{p^{k}} \succ\left(\Sigma_{i} \times \Sigma_{j}\right) \subset \Sigma_{p^{k}} \nmid \Sigma_{p^{l}}$, where $i+j=p^{l}$ and $i, j>0$. In both of these cases, these results do not contradict our main result. 


\section{REFERENCES}

[Ada78] J. F. Adams. Maps between classifying spaces. II. Invent. Math., 49(1):1-65, 1978. (cit. on p. 2).

[AHS04] Matthew Ando, Michael J. Hopkins, and Neil P. Strickland. The sigma orientation is an $H_{\infty}$ map. Amer. J. Math., 126(2):247-334, 2004. (cit. on p. 1).

[BS16] Tobias Barthel and Nathaniel Stapleton. Centralizers in good groups are good. Algebr. Geom. Topol., 16(3):1453-1472, 2016. (cit on pp. 2, 3, 4).

[BS17] Tobias Barthel and Nathaniel Stapleton. Brown-Peterson cohomology from Morava E-theory. Compos. Math., 153(4):780-819, 2017. With an appendix by Jeremy Hahn. (cit. on p. 4).

[Dri74] V. G. Drinfel'd. Elliptic modules. Mat. Sb. (N.S.), 94(136):594-627, 656, 1974. (cit. on p. 1).

[GS99] J. P. C. Greenlees and N. P. Strickland. Varieties and local cohomology for chromatic group cohomology rings. Topology, 38(5):1093-1139, 1999. (cit. on p. 3).

[HKR00] Michael J. Hopkins, Nicholas J. Kuhn, and Douglas C. Ravenel. Generalized group characters and complex oriented cohomology theories. J. Am. Math. Soc., 13(3):553-594, 2000. (cit on pp. 2, 3).

[HS20] Zhen Huan and Nathaniel Stapleton. Level structures on $p$-divisible groups from the Morava E-theory of abelian groups. arXiv e-prints, page arXiv:2001.10075, Jan 2020. (cit on pp. 2, 4).

[Nel18] Peter Nelson. On the Morava E-theory of wreath products of symmetric groups. arXiv e-prints, page arXiv:1803.05020, Mar 2018. (cit. on p. 4).

[Rez09] Charles Rezk. The congruence criterion for power operations in Morava E-theory. Homology Homotopy Appl., 11(2):327-379, 2009. (cit. on p. 1).

[Rez12] Charles Rezk. Rings of power operations for Morava E-theories are Koszul. arXiv e-prints, page arXiv:1204.4831, Apr 2012. (cit. on p. 1).

[SS15] Tomer M. Schlank and Nathaniel Stapleton. A transchromatic proof of Strickland's theorem. Adv. Math., 285:1415-1447, 2015. (cit. on p. 4).

[Sta13] Nathaniel Stapleton. Transchromatic generalized character maps. Algebr. Geom. Topol., 13(1):171-203, 2013. (cit. on p. 3).

[Sta15] Nathaniel Stapleton. Transchromatic twisted character maps. J. Homotopy Relat. Struct., 10(1):29-61, 2015. (cit. on p. 3).

[Str98] N. P. Strickland. Morava E-theory of symmetric groups. Topology, 37(4):757-779, 1998. (cit on pp. 1, 4).

Max Planck Institute for Mathematics, Vivatsgasse 7, 53111 Bonn, Germany

E-mail address: tbarthel@mpim-bonn.mpg.de

University of Kentucky, LeXington, Kentucky, USA

E-mail address: nat.j.stapleton@uky.edu 